\title{
PERIODIC SOLUTIONS OF A DELAYED PREDATOR-PREY MODEL WITH STAGE STRUCTURE FOR PREDATOR
}

\author{
RUI XU, M. A. J. CHAPLAIN, AND F. A. DAVIDSON \\ Received 28 August 2003 and in revised form 2 February 2004
}

A periodic time-dependent Lotka-Volterra-type predator-prey model with stage structure for the predator and time delays due to negative feedback and gestation is investigated. Sufficient conditions are derived, respectively, for the existence and global stability of positive periodic solutions to the proposed model.

\section{Introduction}

The traditional Lotka-Volterra-type predator-prey model has received great attention from both theoretical and mathematical biologists, and has been well studied (see, e.g., $[5,8,9])$. It is assumed in the classical Lotka-Volterra predator-prey model that each individual predator admits the same ability to attack prey. This assumption seems not to be realistic for many animals. In the natural world, there are many species whose individuals have a life history that takes them through two stages, immature and mature. Stagestructured models have received much attention in recent years. In [1], a stage-structured model of single species growth consisting of immature and mature individuals was proposed and discussed. In [2], it was further assumed that the time from immaturity to maturity is itself state-dependent. An equilibrium analysis and eventual lower and upper bounds of positive solutions for the model were addressed. Recently, Wang and Chen [11] proposed a predator-prey model with stage structure for the predator to analyze the influence of a stage structure for the predator on the dynamics of predator-prey models. In [11], the authors classify individuals of the predator as belonging to either the immature or the mature and suppose that the immature predator does not have ability to attack prey. This seems reasonable for a number of mammals, where the immature predators are raised by their parents; the rate they attack prey can be ignored. Sufficient conditions are derived in [11] for the uniform persistence and global stability of a positive equilibrium of the proposed model.

We note that any biological or environmental parameters are naturally subject to fluctuation in time. As Cushing [3] pointed out, it is necessary and important to consider models with periodic ecological parameters or perturbations which might be quite 
naturally exposed (e.g., those due to seasonal effects of weather, food supply, mating habits, hunting or harvesting seasons, etc.). Thus, the assumption of periodicity of the parameters is a way of incorporating the periodicity of the environment.

Motivated by the work of Wang and Chen [11], in the present paper, we incorporate periodicity of the ecological and environmental parameters and stage structure into a Lotka-Volterra-type predator-prey system. To do so, we consider the following delayed periodic time-dependent differential system:

$$
\begin{aligned}
\dot{x}(t) & =x(t)\left(r(t)-a(t) x\left(t-\tau_{1}\right)-a_{1}(t) y_{2}(t)\right), \\
\dot{y}_{1}(t) & =a_{2}(t) x\left(t-\tau_{2}\right) y_{2}\left(t-\tau_{2}\right)-r_{1}(t) y_{1}(t)-b_{1}(t) y_{1}(t), \\
\dot{y}_{2}(t) & =b_{1}(t) y_{1}(t)-r_{2}(t) y_{2}(t),
\end{aligned}
$$

where $x(t)$ represents the density of prey population at time $t, y_{1}(t)$ denotes the density of immature individual predators at time $t, y_{2}(t)$ stands for the density of mature individual predators at time $t, r(t)$ is the intrinsic growth rate of the prey, $a(t)$ is the intraspecific competition rate of the prey, $a_{1}(t)$ is the capturing rate of the mature predator, $a_{2}(t) / a_{1}(t)$ is the conversion rate of nutrients into the reproduction of the predator, $r_{1}(t)$ is the death rate of the immature predator $r_{2}(t)$ the death rate of the mature predator, and $b_{1}(t)$ denotes the rate of immature predator becoming mature predator. It is assumed that this rate is proportional to the density of the existing immature predators. $\tau_{1} \geq 0$ and $\tau_{2} \geq 0$ are constant delays. $\tau_{1}$ represents the time delay due to negative feedback of the prey population, and $\tau_{2}$ denotes the time delay due to gestation of the mature predator.

The initial conditions for system (1.1) take the form of

$$
\begin{gathered}
x(\theta)=\phi(\theta), \quad y_{1}(\theta)=\psi_{1}(\theta), \quad y_{2}(\theta)=\psi_{2}(\theta), \\
\phi(\theta) \geq 0, \quad \psi_{1}(\theta) \geq 0, \quad \psi_{2}(\theta) \geq 0, \quad \theta \in[-\tau, 0], \\
\phi(0)>0, \quad \psi_{1}(0)>0, \quad \psi_{2}(0)>0,
\end{gathered}
$$

where $\tau=\max \left\{\tau_{1}, \tau_{2}\right\}, \Phi=\left(\phi(\theta), \psi_{1}(\theta), \psi_{2}(\theta)\right) \in C\left([-\tau, 0], R_{+0}^{3}\right)$, the Banach space of continuous functions mapping the interval $[-\tau, 0]$ into $R_{+0}^{3}$, where

$$
R_{+0}^{3}=\left\{\left(x_{1}, x_{2}, x_{3}\right): x_{i} \geq 0, i=1,2,3\right\} .
$$

In this paper, for system (1.1), we always assume that the following holds.

(H1) $r(t), a(t), a_{1}(t), a_{2}(t), b_{1}(t), r_{1}(t)$, and $r_{2}(t)$ are continuously positive periodic functions with period $\omega$.

It is well known by the fundamental theory of functional differential equations [7] that system (1.1) has a unique solution $X(t)=\left(x(t), y_{1}(t), y_{2}(t)\right)$ satisfying initial conditions (1.2). It is easy to show that all solutions of system (1.1) corresponding to initial conditions (1.2) are defined on $[0,+\infty)$ and remain positive for all $t \geq 0$. In this paper, the solution of system (1.1) satisfying initial conditions (1.2) is said to be positive.

The organization of this paper is as follows. In Section 2, by using Gaines and Mawhin's continuation theorem of coincidence degree theory [4], sufficient conditions are derived for the existence of positive periodic solutions to system (1.1) with initial conditions (1.2). 
By proving the permanence of system (1.1) and by using an existence result from Teng and Chen [10], we establish a set of different sufficient conditions for the existence of positive periodic solutions of system (1.1). In Section 3, by constructing a suitable Lyapunov functional, sufficient conditions are obtained to guarantee that if system (1.1) has a positive periodic solution, then it is globally stable. A brief discussion is given in Section 4 to conclude this work.

\section{Existence of periodic solutions}

The objective of this section is to derive sufficient conditions for the existence of positive periodic solutions to system (1.1) with initial conditions (1.2).

We first give a result on the existence of positive periodic solutions of system (1.1) by using Gaines and Mawhin's continuation theorem of coincidence degree theory. We begin by presenting certain concepts and notations which will be basic for this section.

Let $X, Y$ be real Banach spaces, $L:$ Dom $L \subset X \rightarrow Y$ a linear mapping, and $N: X \rightarrow Y$ a continuous mapping. The mapping $L$ is called a Fredholm mapping of index zero if $\operatorname{dim} \operatorname{Ker} L=\operatorname{codim} \operatorname{Im} L<+\infty$ and $\operatorname{Im} L$ is closed in $Y$. If $L$ is a Fredholm mapping of index zero and there exist continuous projectors $P: X \rightarrow X$ and $Q: Y \rightarrow Y$ such that $\operatorname{Im} P=\operatorname{Ker} L, \operatorname{Ker} Q=\operatorname{Im} L=\operatorname{Im}(I-Q)$, then the restriction $L_{P}$ of $L$ to $\operatorname{Dom} L \cap \operatorname{Ker} P$ : $(I-P) X \rightarrow \operatorname{Im} L$ is invertible. Denote the inverse of $L_{P}$ by $K_{P}$. If $\Omega$ is an open bounded subset of $X$, the mapping $N$ will be called $L$-compact on $\bar{\Omega}$ if $Q N(\bar{\Omega})$ is bounded and $K_{P}(I-Q) N: \bar{\Omega} \rightarrow X$ is compact. Since $\operatorname{Im} Q$ is isomorphic to $\operatorname{Ker} L$, there exists an isomorphism $J: \operatorname{Im} Q \rightarrow \operatorname{Ker} L$.

For convenience of use, we introduce the continuation theorem of coincidence degree theory (see Gaines and Mawhin [4, page 40]) as follows.

Lemma 2.1. Let $\Omega \subset X$ be an open bounded set. Let $L$ be a Fredholm mapping of index zero and let $N$ be L-compact on $\bar{\Omega}$. Assume

(a) for each $\lambda \in(0,1), x \in \partial \Omega \cap \operatorname{Dom} L, L x \neq \lambda N x$;

(b) for each $x \in \partial \Omega \cap \operatorname{Ker} L, Q N x \neq 0$;

(c) $\operatorname{deg}\{J Q N, \Omega \cap \operatorname{Ker} L, 0\} \neq 0$.

Then $L x=N x$ has at least one solution in $\bar{\Omega} \cap \operatorname{Dom} L$.

In what follows, we will use the following notations:

$$
\bar{f}=\frac{1}{\omega} \int_{0}^{\omega} f(t) d t, \quad f^{L}=\min _{t \in[0, \omega]} f(t), \quad f^{M}=\max _{[0, \omega]} f(t),
$$

where $f$ is a continuous $\omega$-periodic function.

We are now able to state and prove our result on the existence of positive periodic solutions to system (1.1) with initial conditions (1.2).

THEOREM 2.2. In addition to (H1), assume further that

(H2) $\bar{r} a_{2}^{L} b_{1}^{L}-\bar{a} r_{2}^{M}\left(r_{1}^{M}+b_{1}^{M}\right) e^{2 \bar{r} \omega}>0$.

Then system (1.1) with initial conditions (1.2) admits at least one positive w-periodic solution. 
Proof. Let

$$
u_{1}(t)=\ln [x(t)], \quad u_{2}(t)=\ln \left[y_{1}(t)\right], \quad u_{3}(t)=\ln \left[y_{2}(t)\right] .
$$

On substituting (2.2) into (1.1), we derive

$$
\begin{aligned}
& \frac{d u_{1}(t)}{d t}=r(t)-a(t) e^{u_{1}\left(t-\tau_{1}\right)}-a_{1}(t) e^{u_{3}(t)}, \\
& \frac{d u_{2}(t)}{d t}=a_{2}(t) e^{u_{1}\left(t-\tau_{2}\right)+u_{3}\left(t-\tau_{2}\right)-u_{2}(t)}-r_{1}(t)-b_{1}(t), \\
& \frac{d u_{3}(t)}{d t}=b_{1}(t) e^{u_{2}(t)-u_{3}(t)}-r_{2}(t) .
\end{aligned}
$$

It is easy to see that if system $(2.3)$ has one $\omega$-periodic solution $\left(u_{1}^{*}(t), u_{2}^{*}(t), u_{3}^{*}(t)\right)^{T}$, then $X^{*}(t)=\left(x^{*}(t), y_{1}^{*}(t), y_{2}^{*}(t)\right)^{T}=\left(\exp \left[u_{1}^{*}(t)\right], \exp \left[u_{2}^{*}(t)\right], \exp \left[u_{3}^{*}(t)\right]\right)^{T}$ is a positive $\omega$-periodic solution of system (1.1). Hence, to complete the proof, it suffices to show that system (2.3) has at least one $\omega$-periodic solution.

Take

$$
\begin{gathered}
X=Y=\left\{\left(u_{1}(t), u_{2}(t), u_{3}(t)\right)^{T} \in C\left(R, R^{3}\right): u_{i}(t+\omega)=u_{i}(t), i=1,2,3\right\}, \\
\left\|\left(u_{1}(t), u_{2}(t), u_{3}(t)\right)^{T}\right\|=\sum_{i=1}^{3} \max _{t \in[0, \omega]}\left|u_{i}(t)\right|
\end{gathered}
$$

here $|\cdot|$ denotes the Euclidean norm. It is easy to see that $X$ and $Y$ are Banach spaces. Let

$$
L: \operatorname{Dom} L \cap X \rightarrow X, \quad L\left(u_{1}(t), u_{2}(t), u_{3}(t)\right)^{T}=\left(\frac{d u_{1}(t)}{d t}, \frac{d u_{2}(t)}{d t}, \frac{d u_{3}(t)}{d t}\right)^{T},
$$

where $\operatorname{Dom} L=\left\{\left(u_{1}(t), u_{2}(t), u_{3}(t)\right)^{T} \in C^{1}\left(R, R^{3}\right)\right\}$ and $N: X \rightarrow X$,

$$
N\left[\begin{array}{l}
u_{1} \\
u_{2} \\
u_{3}
\end{array}\right]=\left[\begin{array}{c}
r(t)-a(t) e^{u_{1}\left(t-\tau_{1}\right)}-a_{1}(t) e^{u_{3}(t)} \\
a_{2}(t) e^{u_{1}\left(t-\tau_{2}\right)+u_{3}\left(t-\tau_{2}\right)-u_{2}(t)}-r_{1}(t)-b_{1}(t) \\
b_{1}(t) e^{u_{2}(t)-u_{3}(t)}-r_{2}(t)
\end{array}\right] .
$$

Define

$$
P\left[\begin{array}{l}
u_{1} \\
u_{2} \\
u_{3}
\end{array}\right]=Q\left[\begin{array}{l}
u_{1} \\
u_{2} \\
u_{3}
\end{array}\right]=\left[\begin{array}{l}
\frac{1}{\omega} \int_{0}^{\omega} u_{1}(t) d t \\
\frac{1}{\omega} \int_{0}^{\omega} u_{2}(t) d t \\
\frac{1}{\omega} \int_{0}^{\omega} u_{3}(t) d t
\end{array}\right], \quad\left[\begin{array}{l}
u_{1} \\
u_{2} \\
u_{3}
\end{array}\right] \in X=Y .
$$

It is clear that

$$
\begin{aligned}
\operatorname{Ker} L & =\left\{x \mid x \in X, x=h, h \in R^{3}\right\}, \\
\operatorname{Im} L & =\left\{y \mid y \in Y, \int_{0}^{\omega} y(t) d t=0\right\} \quad \text { is closed in } Y, \\
\operatorname{dim} \operatorname{Ker} L & =\operatorname{codim} \operatorname{Im} L=3 .
\end{aligned}
$$


Therefore, $L$ is a Fredholm mapping of index zero. Obviously, $P$ and $Q$ are continuous projectors such that

$$
\operatorname{Im} P=\operatorname{Ker} L, \quad \operatorname{Ker} Q=\operatorname{Im} L=\operatorname{Im}(I-Q) .
$$

Furthermore, it is easy to prove that the inverse $K_{P}$ of $L_{P}$ exists and has the form $\operatorname{Im} L \rightarrow$ $\operatorname{Dom} L \cap \operatorname{Ker} P$,

$$
K_{P}(y)=\int_{0}^{t} y(s) d s-\frac{1}{\omega} \int_{0}^{\omega} \int_{0}^{t} y(s) d s d t
$$

Then $Q N: X \rightarrow Y$ and $K_{P}(I-Q) N: X \rightarrow X$ are given by

$$
\begin{aligned}
& Q N x=\left[\begin{array}{c}
\frac{1}{\omega} \int_{0}^{\omega}\left[r(t)-a(t) e^{u_{1}\left(t-\tau_{1}\right)}-a_{1}(t) e^{u_{3}(t)}\right] d t \\
\frac{1}{\omega} \int_{0}^{\omega}\left[a_{2}(t) e^{u_{1}\left(t-\tau_{2}\right)+u_{3}\left(t-\tau_{2}\right)-u_{2}(t)}-r_{1}(t)-b_{1}(t)\right] d t \\
\frac{1}{\omega} \int_{0}^{\omega}\left[b_{1}(t) e^{u_{2}(t)-u_{3}(t)}-r_{2}(t)\right] d t
\end{array}\right], \\
& K_{P}(I-Q) N x=\int_{0}^{t} N x(s) d s-\frac{1}{\omega} \int_{0}^{\omega} \int_{0}^{t} N x(s) d s d t-\left(\frac{t}{\omega}-\frac{1}{2}\right) \int_{0}^{\omega} N x(s) d s .
\end{aligned}
$$

It is easy to see that $Q N$ and $K_{P}(I-Q) N$ are continuous.

In order to apply Lemma 2.1, we need to search for an appropriate open and bounded subset $\Omega$.

Corresponding to the operator equation $L x=\lambda N x, \lambda \in(0,1)$, it follows that

$$
\begin{aligned}
& \frac{d u_{1}(t)}{d t}=\lambda\left[r(t)-a(t) e^{u_{1}\left(t-\tau_{1}\right)}-a_{1}(t) e^{u_{3}(t)}\right] \\
& \frac{d u_{2}(t)}{d t}=\lambda\left[a_{2}(t) e^{u_{1}\left(t-\tau_{2}\right)+u_{3}\left(t-\tau_{2}\right)-u_{2}(t)}-r_{1}(t)-b_{1}(t)\right] \\
& \frac{d u_{3}(t)}{d t}=\lambda\left[b_{1}(t) e^{u_{2}(t)-u_{3}(t)}-r_{2}(t)\right]
\end{aligned}
$$

Suppose that $\left(u_{1}(t), u_{2}(t), u_{3}(t)\right)^{T} \in X$ is a solution of $(2.12)$ for a certain $\lambda \in(0,1)$. Integrating $(2.12)$ over the interval $[0, \omega]$ leads to

$$
\begin{gathered}
\int_{0}^{\omega} a(t) e^{u_{1}\left(t-\tau_{1}\right)} d t+\int_{0}^{\omega} a_{1}(t) e^{u_{3}(t)} d t=\int_{0}^{\omega} r(t) d t \\
\int_{0}^{\omega} a_{2}(t) e^{u_{1}\left(t-\tau_{2}\right)+u_{3}\left(t-\tau_{2}\right)-u_{2}(t)} d t=\int_{0}^{\omega}\left(r_{1}(t)+b_{1}(t)\right) d t \\
\int_{0}^{\omega} b_{1}(t) e^{u_{2}(t)-u_{3}(t)} d t=\int_{0}^{\omega} r_{2}(t) d t .
\end{gathered}
$$


It follows from (2.12), (2.13), (2.14), and (2.15) that

$$
\begin{aligned}
& \int_{0}^{\omega}\left|u_{1}^{\prime}(t)\right| d t<\int_{0}^{\omega}\left[r(t)+a(t) e^{u_{1}\left(t-\tau_{1}\right)}+a_{1}(t) e^{u_{3}(t)}\right] d t=2 \bar{r} \omega, \\
& \int_{0}^{\omega}\left|u_{2}^{\prime}(t)\right| d t< \int_{0}^{\omega}\left[a_{2}(t) e^{u_{1}\left(t-\tau_{2}\right)+u_{3}\left(t-\tau_{2}\right)-u_{2}(t)}+r_{1}(t)+b_{1}(t)\right] d t=2 \omega\left(\bar{r}_{1}+\bar{b}_{1}\right), \\
& \int_{0}^{\omega}\left|u_{3}^{\prime}(t)\right| d t<\int_{0}^{\omega}\left[b_{1}(t) e^{u_{2}(t)-u_{3}(t)}+r_{2}(t)\right] d t=2 \bar{r}_{2} \omega .
\end{aligned}
$$

Since $\left(u_{1}(t), u_{2}(t), u_{3}(t)\right)^{T} \in X$, there exist $\xi_{i}, \eta_{i} \in[0, \omega]$ such that

$$
u_{i}\left(\xi_{i}\right)=\min _{t \in[0, \omega]} u_{i}(t), \quad u_{i}\left(\eta_{i}\right)=\max _{t \in[0, \omega]} u_{i}(t), \quad i=1,2,3
$$

Multiplying the second equation of $(2.12)$ by $e^{u_{2}(t)}$ and integrating over $[0, \omega]$ gives

$$
\int_{0}^{\omega} a_{2}(t) e^{u_{1}\left(t-\tau_{2}\right)+u_{3}\left(t-\tau_{2}\right)} d t=\int_{0}^{\omega}\left(r_{1}(t)+b_{1}(t)\right) e^{u_{2}(t)} d t .
$$

Multiplying the third equation of (2.12) by $e^{u_{3}(t)}$ and integrating over $[0, \omega]$ yields

$$
\int_{0}^{\omega} b_{1}(t) e^{u_{2}(t)} d t=\int_{0}^{\omega} r_{2}(t) e^{u_{3}(t)} d t
$$

We derive from (2.21) that

$$
\begin{aligned}
& \int_{0}^{\omega} e^{u_{2}(t)} d t \leq \frac{r_{2}^{M}}{b_{1}^{L}} \int_{0}^{\omega} e^{u_{3}(t)} d t \\
& \int_{0}^{\omega} e^{u_{2}(t)} d t \geq \frac{r_{2}^{L}}{b_{1}^{M}} \int_{0}^{\omega} e^{u_{3}(t)} d t .
\end{aligned}
$$

It follows from (2.20) and (2.22) that

$$
a_{2}^{L} \int_{0}^{\omega} e^{u_{1}\left(\xi_{1}\right)+u_{3}\left(t-\tau_{2}\right)} d t \leq\left(r_{1}^{M}+b_{1}^{M}\right) \int_{0}^{\omega} e^{u_{2}(t)} d t \leq \frac{r_{2}^{M}\left(r_{1}^{M}+b_{1}^{M}\right)}{b_{1}^{L}} \int_{0}^{\omega} e^{u_{3}(t)} d t .
$$

Noting that

$$
\int_{0}^{\omega} e^{u_{3}\left(t-\tau_{2}\right)} d t=\int_{0}^{\omega} e^{u_{3}(t)} d t
$$

we derive from (2.24) that

$$
u_{1}\left(\xi_{1}\right) \leq \ln \frac{r_{2}^{M}\left(r_{1}^{M}+b_{1}^{M}\right)}{a_{2}^{L} b_{1}^{L}}
$$


It follows from (2.16) and (2.26) that

$$
u_{1}(t) \leq u_{1}\left(\xi_{1}\right)+\int_{0}^{\omega}\left|u_{1}^{\prime}(t)\right| d t \leq \ln \frac{r_{2}^{M}\left(r_{1}^{M}+b_{1}^{M}\right)}{a_{2}^{L} b_{1}^{L}}+2 \bar{r} \omega .
$$

We derive from (2.20) and (2.23) that

$$
a_{2}^{M} \int_{0}^{\omega} e^{u_{1}\left(\eta_{1}\right)+u_{3}\left(t-\tau_{2}\right)} d t \geq\left(r_{1}^{L}+b_{1}^{L}\right) \int_{0}^{\omega} e^{u_{2}(t)} d t \geq \frac{r_{2}^{L}\left(r_{1}^{L}+b_{1}^{L}\right)}{b_{1}^{M}} \int_{0}^{\omega} e^{u_{3}(t)} d t,
$$

which, together with (2.25), leads to

$$
u_{1}\left(\eta_{1}\right) \geq \ln \frac{r_{2}^{L}\left(r_{1}^{L}+b_{1}^{L}\right)}{a_{2}^{M} b_{1}^{M}} .
$$

It follows from (2.16) and (2.29) that

$$
u_{1}(t) \geq u_{1}\left(\eta_{1}\right)-\int_{0}^{\omega}\left|u_{1}^{\prime}(t)\right| d t \geq \ln \frac{r_{2}^{L}\left(r_{1}^{L}+b_{1}^{L}\right)}{a_{2}^{M} b_{1}^{M}}-2 \bar{r} \omega
$$

which, together with (2.27), yields

$$
\max _{t \in[0, \omega]}\left|u_{1}(t)\right|<\max \left\{\left|\ln \frac{r_{2}^{M}\left(r_{1}^{M}+b_{1}^{M}\right)}{a_{2}^{L} b_{1}^{L}}\right|+2 \bar{r} \omega,\left|\ln \frac{r_{2}^{L}\left(r_{1}^{L}+b_{1}^{L}\right)}{a_{2}^{M} b_{1}^{M}}\right|+2 \bar{r} \omega\right\}:=R_{1} .
$$

It follows from (2.13) that

$$
u_{3}\left(\xi_{3}\right) \leq \ln \frac{\bar{r}}{\bar{a}_{1}}
$$

which, together with (2.18), leads to

$$
u_{3}(t) \leq u_{3}\left(\xi_{3}\right)+\int_{0}^{\omega}\left|u_{3}^{\prime}(t)\right| d t \leq \ln \frac{\bar{r}}{\bar{a}_{1}}+2 \bar{r}_{2} \omega .
$$

We derive from (2.13) and (2.27) that

$$
\begin{aligned}
a_{1}^{M} \omega e^{u_{3}\left(\eta_{3}\right)} & \geq \bar{r} \omega-\int_{0}^{\omega} a(t) e^{u_{1}\left(t-\tau_{1}\right)} d t \\
& \geq \bar{r} \omega-\int_{0}^{\omega} a(t) \exp \left\{\ln \frac{r_{2}^{M}\left(r_{1}^{M}+b_{1}^{M}\right)}{a_{2}^{L} b_{1}^{L}}+2 \bar{r} \omega\right\} d t \\
& =\bar{r} \omega-\frac{\bar{a} r_{2}^{M} \omega\left(r_{1}^{M}+b_{1}^{M}\right)}{a_{2}^{L} b_{1}^{L}} e^{2 \bar{r} \omega},
\end{aligned}
$$

which yields

$$
u_{3}\left(\eta_{3}\right) \geq \ln \frac{\bar{r} a_{2}^{L} b_{1}^{L}-\bar{a} r_{2}^{M}\left(r_{1}^{M}+b_{1}^{M}\right) e^{2 \bar{r} \omega}}{a_{1}^{M} a_{2}^{L} b_{1}^{L}}
$$


262 A predator-prey model with stage structure

which, together with (2.18), leads to

$$
u_{3}(t) \geq u_{3}\left(\eta_{3}\right)-\int_{0}^{\omega}\left|u_{3}^{\prime}(t)\right| d t \geq \ln \frac{\bar{r} a_{2}^{L} b_{1}^{L}-\bar{a} r_{2}^{M}\left(r_{1}^{M}+b_{1}^{M}\right) e^{2 \bar{r} \omega}}{a_{1}^{M} a_{2}^{L} b_{1}^{L}}-2 \bar{r}_{2} \omega .
$$

It follows from (2.33) and (2.36) that

$$
\max _{t \in[0, \omega]}\left|u_{3}(t)\right|<\max \left\{\left|\ln \frac{\bar{r}}{\bar{a}_{1}}\right|+2 \bar{r}_{2} \omega,\left|\ln \frac{\bar{r} a_{2}^{L} b_{1}^{L}-\bar{a} r_{2}^{M}\left(r_{1}^{M}+b_{1}^{M}\right) e^{2 \bar{r} \omega}}{a_{1}^{M} a_{2}^{L} b_{1}^{L}}\right|+2 \bar{r}_{2} \omega\right\}:=R_{3} .
$$

We derive from (2.22) and (2.33) that

$$
\int_{0}^{\omega} e^{u_{2}(t)} d t \leq \frac{r_{2}^{M}}{b_{1}^{L}} \int_{0}^{\omega} \exp \left\{\ln \frac{\bar{r}}{\bar{a}_{1}}+2 \bar{r}_{2} \omega\right\} d t=\frac{\bar{r} r_{2}^{M} \omega}{\bar{a}_{1} b_{1}^{L}} e^{2 \bar{r}_{2} \omega}
$$

which yields

$$
u_{2}\left(\xi_{2}\right) \leq \ln \frac{\bar{r} r_{2}^{M}}{\bar{a}_{1} b_{1}^{L}}+2 \bar{r}_{2} \omega
$$

It follows from (2.17) and (2.39) that

$$
u_{2}(t) \leq u_{2}\left(\xi_{2}\right)+\int_{0}^{\omega}\left|u_{2}^{\prime}(t)\right| d t \leq \ln \frac{\bar{r} r_{2}^{M}}{\bar{a}_{1} b_{1}^{L}}+2 \omega\left(\bar{r}_{1}+\bar{r}_{2}+\bar{b}_{1}\right)
$$

Similarly, from (2.23) and (2.36), we obtain

$$
\begin{aligned}
\int_{0}^{\omega} e^{u_{2}(t)} d t & \geq \frac{r_{2}^{L}}{b_{1}^{M}} \int_{0}^{\omega} \exp \left\{\ln \frac{\bar{r} a_{2}^{L} b_{1}^{L}-\bar{a} r_{2}^{M}\left(r_{1}^{M}+b_{1}^{M}\right) e^{2 \bar{r} \omega}}{a_{1}^{M} a_{2}^{L} b_{1}^{L}}-2 \bar{r}_{2} \omega\right\} d t \\
& =\frac{r_{2}^{L} \omega\left[\bar{r} a_{2}^{L} b_{1}^{L}-\bar{a} r_{2}^{M}\left(r_{1}^{M}+b_{1}^{M}\right) e^{2 \bar{r} \omega}\right]}{a_{1}^{M} a_{2}^{L} b_{1}^{L} b_{1}^{M}} e^{-2 \bar{r}_{2} \omega},
\end{aligned}
$$

which implies

$$
u_{2}\left(\eta_{2}\right) \geq \ln \frac{r_{2}^{L}\left[\bar{r} a_{2}^{L} b_{1}^{L}-\bar{a} r_{2}^{M}\left(r_{1}^{M}+b_{1}^{M}\right) e^{2 \bar{r} \omega}\right]}{a_{1}^{M} a_{2}^{L} b_{1}^{L} b_{1}^{M}}-2 \bar{r}_{2} \omega
$$

It follows from (2.17) and (2.42) that

$$
\begin{aligned}
u_{2}(t) & \geq u_{2}\left(\eta_{2}\right)-\int_{0}^{\omega}\left|u_{2}^{\prime}(t)\right| d t \\
& \geq \ln \frac{r_{2}^{L}\left[\bar{r} a_{2}^{L} b_{1}^{L}-\bar{a} r_{2}^{M}\left(r_{1}^{M}+b_{1}^{M}\right) e^{2 \bar{r} \omega}\right]}{a_{1}^{M} a_{2}^{L} b_{1}^{L} b_{1}^{M}}-2 \omega\left(\bar{r}_{1}+\bar{r}_{2}+\bar{b}_{1}\right),
\end{aligned}
$$


which, together with (2.40), leads to

$$
\begin{aligned}
& \max _{t \in[0, \omega]}\left|u_{2}(t)\right|<\max \left\{\left|\ln \frac{\bar{r} r_{2}^{M}}{\bar{a}_{1} b_{1}^{L}}\right|+2 \omega\left(\bar{r}_{1}+\bar{r}_{2}+\bar{b}_{1}\right),\right. \\
&\left.\left|\ln \frac{r_{2}^{L}\left[\bar{r} a_{2}^{L} b_{1}^{L}-\bar{a} r_{2}^{M}\left(r_{1}^{M}+b_{1}^{M}\right) e^{2 \bar{r} \omega}\right]}{a_{1}^{M} a_{2}^{L} b_{1}^{L} b_{1}^{M}}\right|+2 \omega\left(\bar{r}_{1}+\bar{r}_{2}+\bar{b}_{1}\right)\right\}:=R_{2} .
\end{aligned}
$$

We note that $R_{1}, R_{2}$, and $R_{3}$ in (2.31), (2.44), and (2.37), respectively, are independent of $\lambda$. Denote $M=R_{1}+R_{2}+R_{3}+R_{0}$, where $R_{0}$ is taken sufficiently large such that the unique solution $\left(\alpha^{*}, \beta^{*}, \gamma^{*}\right)^{T}$ of the algebraic equations

$$
\bar{r}-\bar{a} e^{\alpha}-\bar{a}_{1} e^{\gamma}=0, \quad \bar{a}_{2} e^{\alpha+\gamma-\beta}-\bar{r}_{1}-\bar{b}_{1}=0, \quad \bar{b}_{1} e^{\beta-\gamma}-\bar{r}_{2}=0
$$

satisfies $\left\|\left(\alpha^{*}, \beta^{*}, \gamma^{*}\right)^{T}\right\|=\left|\alpha^{*}\right|+\left|\beta^{*}\right|+\left|\gamma^{*}\right|<M$.

We now take $\Omega=\left\{\left(u_{1}(t), u_{2}(t), u_{3}(t)\right)^{T} \in X:\left\|\left(u_{1}, u_{2}, u_{3}\right)^{T}\right\|<M\right\}$. This satisfies Lemma 2.1(a). When $\left(u_{1}(t), u_{2}(t), u_{3}(t)\right)^{T} \in \partial \Omega \cap \operatorname{Ker} L=\partial \Omega \cap R^{3},\left(u_{1}, u_{2}, u_{3}\right)^{T}$ is a constant vector in $R^{3}$ with $\left|u_{1}\right|+\left|u_{2}\right|+\left|u_{3}\right|=M$. Thus, we have

$$
Q N\left[\begin{array}{l}
u_{1} \\
u_{2} \\
u_{3}
\end{array}\right]=\left[\begin{array}{c}
\bar{r}-\bar{a} e^{u_{1}}-\bar{a}_{1} e^{u_{3}} \\
\bar{a}_{2} e^{u_{1}+u_{3}-u_{2}}-\bar{r}_{1}-\bar{b}_{1} \\
\bar{b}_{1} e^{u_{2}-u_{3}}-\bar{r}_{2}
\end{array}\right] \neq\left[\begin{array}{l}
0 \\
0 \\
0
\end{array}\right] .
$$

This proves that Lemma 2.1(b) is satisfied.

We now prove that Lemma 2.1(c) holds. Taking $J=I: \operatorname{Im} Q \rightarrow \operatorname{Ker} L,\left(u_{1}, u_{2}, u_{3}\right)^{T} \rightarrow$ $\left(u_{1}, u_{2}, u_{3}\right)^{T}$, and by a direct calculation, we can derive

$$
\begin{aligned}
\operatorname{deg}( & \left.\operatorname{JQN}\left(u_{1}, u_{2}, u_{3}\right)^{T}, \Omega \cap \operatorname{Ker} L,(0,0,0)^{T}\right) \\
= & \operatorname{deg}\left(\left(\bar{r}-\bar{a} e^{u_{1}}-\bar{a}_{1} e^{u_{3}}, \bar{a}_{2} e^{u_{1}+u_{3}-u_{2}}-\bar{r}_{1}-\bar{b}_{1}, \bar{b}_{1} e^{u_{2}-u_{3}}-\bar{r}_{2}\right)^{T},\right. \\
& \left.\quad \Omega \cap \operatorname{Ker} L,(0,0,0)^{T}\right) \\
= & \operatorname{sgn}\left\{-\bar{a}_{1} \bar{a}_{2} \bar{b}_{1} e^{\alpha^{*}+\gamma^{*}}\right\} \\
= & -1,
\end{aligned}
$$

where $\left(\alpha^{*}, \beta^{*}, \gamma^{*}\right)$ is the unique solution of the algebraic equations (2.45).

Finally, it is easy to show that the set $\left\{K_{P}(I-Q) N x \mid x \in \bar{\Omega}\right\}$ is equicontinuous and uniformly bounded. By using the Arzela-Ascoli theorem, we see that $K_{P}(I-Q) N: \bar{\Omega} \rightarrow X$ is compact. Consequently, $N$ is $L$-compact.

By now we have proved that $\Omega$ satisfies all the requirements in Lemma 2.1. Hence, (2.3) has at least one $\omega$-periodic solution. Accordingly, system (1.1) has at least one positive $\omega$ periodic solution. This completes the proof.

We will now proceed to derive a set of different conditions for the existence of positive periodic solutions to system (1.1) by using a different approach. To this end, we first obtain certain upper bound estimates for positive solutions of system (1.1) with initial conditions (1.2). 
Lemma 2.3. Let $\left(x(t), y_{1}(t), y_{2}(t)\right)$ be any positive solution to system (1.1) with initial conditions (1.2). Then for $\varepsilon>0$ sufficiently small, there exists a $T>0$ such that if $t>T, x(t) \leq M_{1}$, $y_{1}(t) \leq M_{2}, y_{2}(t) \leq M_{3}$, where

$$
\begin{aligned}
& M_{1}=\frac{r^{M}}{a^{L}} e^{r^{M} \tau_{1}}, \quad M_{2}=\frac{a_{2}^{M} M_{1}\left(r^{M}+r_{1}^{L}+b_{1}^{L}\right)}{a_{1}^{L}\left(r_{1}^{L}+b_{1}^{L}\right)}+\varepsilon, \\
& M_{3}=\frac{a_{2}^{M} b_{1}^{M} M_{1}\left(r^{M}+r_{1}^{L}+b_{1}^{L}\right)}{a_{1}^{L} r_{2}^{L}\left(r_{1}^{L}+b_{1}^{L}\right)}+\varepsilon .
\end{aligned}
$$

Proof. It follows from the first equation of system (1.1) that

$$
\dot{x}(t) \leq x(t)\left(r^{M}-a^{L} x\left(t-\tau_{1}\right)\right) .
$$

By [12, Lemma 2.2], there is a $T_{1}>0$ such that if $t>T_{1}$,

$$
x(t) \leq \frac{r^{m}}{a^{L}} e^{r^{M} \tau_{1}}:=M_{1} .
$$

Let

$$
\rho(t)=a_{2}^{M} x\left(t-\tau_{2}\right)+a_{1}^{L} y_{1}(t)
$$

Calculating the derivative of $\rho(t)$ along positive solutions of system (1.1), we derive that

$$
\begin{aligned}
\rho^{\prime}(t)= & a_{2}^{M} x\left(t-\tau_{2}\right)\left[r\left(t-\tau_{2}\right)-a\left(t-\tau_{2}\right) x\left(t-\tau_{1}-\tau_{2}\right)-a_{1}\left(t-\tau_{2}\right) y_{2}\left(t-\tau_{2}\right)\right] \\
& +a_{1}^{L} a_{2}(t) x\left(t-\tau_{2}\right) y_{2}\left(t-\tau_{2}\right)-a_{1}^{L}\left(r_{1}(t)+b_{1}(t)\right) y_{1}(t) \\
\leq & a_{2}^{M} r^{M} x\left(t-\tau_{2}\right)-a_{1}^{L}\left(r_{1}^{L}+b_{1}^{L}\right) y_{1}(t) \\
\leq & -\left(r_{1}^{L}+b_{1}^{L}\right) \rho(t)+a_{2}^{M}\left(r^{M}+r_{1}^{L}+b_{1}^{L}\right) x\left(t-\tau_{2}\right),
\end{aligned}
$$

which, together with (2.50), yields

$$
\limsup _{t \rightarrow+\infty} \rho(t) \leq \frac{a_{2}^{M} M_{1}\left(r^{M}+r_{1}^{L}+b_{1}^{L}\right)}{r_{1}^{L}+b_{1}^{L}}
$$

Therefore, for $\varepsilon>0$ sufficiently small, there is a $T_{2}>T_{1}+\tau_{1}+\tau_{2}$ such that if $t>T_{2}$,

$$
y_{1}(t) \leq \frac{a_{2}^{M} M_{1}\left(r^{M}+r_{1}^{L}+b_{1}^{L}\right)}{a_{1}^{L}\left(r_{1}^{L}+b_{1}^{L}\right)}+\varepsilon:=M_{2} .
$$

It follows from the third equation of system (1.1) that for $t>T_{2}$,

$$
\dot{y}_{2}(t) \leq b_{1}^{M} M_{2}-r_{2}^{L} y_{2}(t)
$$


which yields

$$
\limsup _{t \rightarrow+\infty} y_{2}(t) \leq \frac{b_{1}^{M}}{r_{2}^{L}}\left(\frac{a_{2}^{M} M_{1}\left(r^{M}+r_{1}^{L}+b_{1}^{L}\right)}{a_{1}^{L}\left(r_{1}^{L}+b_{1}^{L}\right)}+\varepsilon\right) .
$$

Since $\varepsilon>0$ is arbitrary and sufficiently small, we conclude that

$$
\limsup _{t \rightarrow+\infty} y_{2}(t) \leq \frac{a_{2}^{M} b_{1}^{M} M_{1}\left(r^{M}+r_{1}^{L}+b_{1}^{L}\right)}{a_{1}^{L} r_{2}^{L}\left(r_{1}^{L}+b_{1}^{L}\right)}:=M_{3}^{*} \text {. }
$$

Hence, for $\varepsilon>0$ sufficiently small, there is a $T>T_{2}$ such that if $t>T, y_{2}(t) \leq M_{3}^{*}+\varepsilon:=$ $M_{3}$. This completes the proof.

THEOREM 2.4. System (1.1) has at least one positive periodic solution provided that

(H3) $r^{L} \tau_{1} \leq 3 / 2$,

(H4) $a_{2}^{L} b_{1}^{L} r^{L}>a^{M} r_{2}^{M}\left(b_{1}^{M}+r_{1}^{M}\right)$.

Proof. Let $\left(x(t), y_{1}(t), y_{2}(t)\right)$ be a positive solution of system (1.1) with initial conditions (1.2). It then follows from system (1.1) that

$$
\begin{aligned}
\dot{x}(t) & \geq x(t)\left(r^{L}-a^{M} x\left(t-\tau_{1}\right)-a_{1}^{M} y_{2}(t)\right), \\
\dot{y}_{1}(t) & \geq a_{2}^{L} x\left(t-\tau_{2}\right) y_{2}\left(t-\tau_{2}\right)-r_{1}^{M} y_{1}(t)-b_{1}^{M} y_{1}(t), \\
\dot{y}_{2}(t) & \geq b_{1}^{L} y_{1}(t)-r_{2}^{M} y_{2}(t) .
\end{aligned}
$$

Consider the following auxiliary system:

$$
\begin{aligned}
\dot{x}(t) & =x(t)\left(r^{L}-a^{M} x\left(t-\tau_{1}\right)-a_{1}^{M} y_{2}(t)\right), \\
\dot{y}_{1}(t) & =a_{2}^{L} x\left(t-\tau_{2}\right) y_{2}\left(t-\tau_{2}\right)-r_{1}^{M} y_{1}(t)-b_{1}^{M} y_{1}(t), \\
\dot{y}_{2}(t) & =b_{1}^{L} y_{1}(t)-r_{2}^{M} y_{2}(t) .
\end{aligned}
$$

By similar arguments as in the proof of [11, Theorem 2.1], we can prove that if $(\mathrm{H} 3)$, (H4) hold, system (2.59) is permanent. As a consequence, system (1.1) is permanent. Therefore, by [10, Theorem 2], we obtain that system (1.1) admits at least one positive $\omega$-periodic solution. The proof is complete.

\section{Global stability}

In this section, we are concerned with the global stability of positive periodic solutions to system (1.1) with initial conditions (1.2). The strategy of proof is to construct a suitable Lyapunov functional. 
Theorem 3.1. If system (1.1) has a positive periodic solution, then it is globally stable provided that

(H5) $\liminf _{t \rightarrow \infty} A_{i}(t)>0$,

where

$$
\begin{aligned}
A_{1}(t)= & a(t)-a_{2}\left(t+\tau_{2}\right) M_{3}-a\left(t+\tau_{1}\right) M_{1} \int_{t+\tau_{1}}^{t+2 \tau_{1}} a(s) d s \\
& -M_{1}\left(r(t)+a(t) M_{1}+a_{1}(t) M_{3}\right) \int_{t}^{t+\tau_{1}} a(s) d s \\
A_{2}(t)= & r_{2}(t)-a_{1}(t)-a_{2}\left(t+\tau_{2}\right) M_{1}-a_{1}(t) M_{1} \int_{t}^{t+\tau_{1}} a(s) d s
\end{aligned}
$$

in which $M_{1}$ and $M_{3}$ are defined in (2.48).

Proof. Let $\left(x^{*}(t), y_{1}^{*}(t), y_{2}^{*}(t)\right)^{T}$ be a positive $\omega$-periodic solution of system (1.1) with initial conditions (1.2). Suppose that $\left(x(t), y_{1}(t), y_{2}(t)\right)^{T}$ is any positive solution of system (1.1).

Let

$$
V_{1}(t)=\left|\ln x(t)-\ln x^{*}(t)\right|+\left|y_{1}(t)-y_{1}^{*}(t)\right|+\left|y_{2}(t)-y_{2}^{*}(t)\right|
$$

Calculating the upper-right derivative of $V_{1}(t)$ along positive solutions of (1.1), it follows that

$$
\begin{aligned}
D^{+} V_{1}(t)= & \left(\frac{\dot{x}(t)}{x(t)}-\frac{\dot{x}^{*}(t)}{x^{*}(t)}\right) \operatorname{sgn}\left(x(t)-x^{*}(t)\right)+\sum_{i=1}^{2}\left(\dot{y}_{i}(t)-\dot{y}_{i}^{*}(t)\right) \operatorname{sgn}\left(y_{i}(t)-y_{i}^{*}(t)\right) \\
= & \operatorname{sgn}\left(x(t)-x^{*}(t)\right)\left\{-a(t)\left(x\left(t-\tau_{1}\right)-x^{*}\left(t-\tau_{1}\right)\right)-a_{1}(t)\left(y_{2}(t)-y_{2}^{*}(t)\right)\right\} \\
& +\operatorname{sgn}\left(y_{1}(t)-y_{1}^{*}(t)\right)\left\{a_{2}(t) x\left(t-\tau_{2}\right)\left(y_{2}\left(t-\tau_{2}\right)-y_{2}^{*}\left(t-\tau_{2}\right)\right)\right. \\
& +a_{2}(t) y_{2}^{*}\left(t-\tau_{2}\right)\left(x\left(t-\tau_{2}\right)-x^{*}\left(t-\tau_{2}\right)\right) \\
& \left.-\left(r_{1}(t)+b_{1}(t)\right)\left(y_{1}(t)-y_{1}^{*}(t)\right)\right\} \\
+ & \operatorname{sgn}\left(y_{2}(t)-y_{2}^{*}(t)\right)\left\{b_{1}(t)\left(y_{1}(t)-y_{1}^{*}(t)\right)-r_{2}(t)\left(y_{2}(t)-y_{2}^{*}(t)\right)\right\} \\
= & \operatorname{sgn}\left(x(t)-x^{*}(t)\right)\left\{-a(t)\left(x(t)-x^{*}(t)\right)-a_{1}(t)\left(y_{2}(t)-y_{2}^{*}(t)\right)\right. \\
+ & \left.a(t) \int_{t-\tau_{1}}^{t}\left(\dot{x}(u)-\dot{x}^{*}(u)\right) d u\right\} \\
+ & \operatorname{sgn}\left(y_{1}(t)-y_{1}^{*}(t)\right)\left\{a_{2}(t) x\left(t-\tau_{2}\right)\left(y_{2}\left(t-\tau_{2}\right)-y_{2}^{*}\left(t-\tau_{2}\right)\right)\right. \\
& +a_{2}(t) y_{2}^{*}\left(t-\tau_{2}\right)\left(x\left(t-\tau_{2}\right)-x^{*}\left(t-\tau_{2}\right)\right) \\
- & \left.\left(r_{1}(t)+b_{1}(t)\right)\left(y_{1}(t)-y_{1}^{*}(t)\right)\right\} \\
+ & \operatorname{sgn}\left(y_{2}(t)-y_{2}^{*}(t)\right)\left\{b_{1}(t)\left(y_{1}(t)-y_{1}^{*}(t)\right)-r_{2}(t)\left(y_{2}(t)-y_{2}^{*}(t)\right)\right\} .
\end{aligned}
$$


On substituting (1.1) into (3.3), we derive that

$$
\begin{aligned}
& \begin{array}{l}
D^{+} V_{1}(t) \\
=\operatorname{sgn}\left(x(t)-x^{*}(t)\right)\left\{\begin{array}{l}
-a(t)\left(x(t)-x^{*}(t)\right)-a_{1}(t)\left(y_{2}(t)-y_{2}^{*}(t)\right) \\
+a(t) \int_{t-\tau_{1}}^{t}\left\{x ( u ) \left[-a(u)\left(x\left(u-\tau_{1}\right)-x^{*}\left(u-\tau_{1}\right)\right)\right.\right. \\
\left.-a_{1}(u)\left(y_{2}(u)-y_{2}^{*}(u)\right)\right]
\end{array}\right. \\
\left.\left.+\left(x(u)-x^{*}(u)\right)\left(r(u)-a(u) x^{*}\left(u-\tau_{1}\right)-a_{1}(u) y_{2}^{*}(u)\right)\right\} d u\right\} \\
+\operatorname{sgn}\left(y_{1}(t)-y_{1}^{*}(t)\right)\left\{a_{2}(t) x\left(t-\tau_{2}\right)\left(y_{2}\left(t-\tau_{2}\right)-y_{2}^{*}\left(t-\tau_{2}\right)\right)\right. \\
\quad+a_{2}(t) y_{2}^{*}\left(t-\tau_{2}\right)\left(x\left(t-\tau_{2}\right)-x^{*}\left(t-\tau_{2}\right)\right) \\
\left.-\left(r_{1}(t)+b_{1}(t)\right)\left(y_{1}(t)-y_{1}^{*}(t)\right)\right\}
\end{array} \\
& +\operatorname{sgn}\left(y_{2}(t)-y_{2}^{*}(t)\right)\left\{b_{1}(t)\left(y_{1}(t)-y_{1}^{*}(t)\right)-r_{2}(t)\left(y_{2}(t)-y_{2}^{*}(t)\right)\right\} \\
& \begin{array}{r}
+a(t)\left|x(t)-x^{*}(t)\right|+a_{1}(t)\left|y_{2}(t)-y_{2}^{*}(t)\right| \\
+a(t) \int_{t-\tau_{1}}^{t}\left\{x(u)\left[a(u)\left|x\left(u-\tau_{1}\right)-x^{*}\left(u-\tau_{1}\right)\right|+a_{1}(u)\left|y_{2}(u)-y_{2}^{*}(u)\right|\right]\right. \\
\left.+\left|x(u)-x^{*}(u)\right|\left(r(u)+a(u) x^{*}\left(u-\tau_{1}\right)+a_{1}(u) y_{2}^{*}(u)\right)\right\} d u
\end{array} \\
& +a_{2}(t) x\left(t-\tau_{2}\right)\left|y_{2}\left(t-\tau_{2}\right)-y_{2}^{*}\left(t-\tau_{2}\right)\right|+a_{2}(t) y_{2}^{*}\left(t-\tau_{2}\right)\left|x\left(t-\tau_{2}\right)-x^{*}\left(t-\tau_{2}\right)\right| \\
& -\left(r_{1}(t)+b_{1}(t)\right)\left|y_{1}(t)-y_{1}^{*}(t)\right|+b_{1}(t)\left|y_{1}(t)-y_{1}^{*}(t)\right|-r_{2}(t)\left|y_{2}(t)-y_{2}^{*}(t)\right| .
\end{aligned}
$$

By Lemma 2.3, there is a $T>0$ such that if $t>T$,

$$
x(t) \leq M_{1}, \quad y_{2}(t) \leq M_{3}, \quad x^{*}(t) \leq M_{1}, \quad y_{2}^{*}(t) \leq M_{3} .
$$

It follows from (3.4) and (3.5) that for $t>T+2 \tau$,

$$
\begin{aligned}
D^{+} V_{1}(t) \leq & -a(t)\left|x(t)-x^{*}(t)\right|+a_{1}(t)\left|y_{2}(t)-y_{2}^{*}(t)\right| \\
& +a(t) M_{1} \int_{t-\tau_{1}}^{t}\left\{a(u)\left|x\left(u-\tau_{1}\right)-x^{*}\left(u-\tau_{1}\right)\right|+a_{1}(u)\left|y_{2}(u)-y_{2}^{*}(u)\right|\right. \\
& \left.\quad+\left(r(u)+a(u) M_{1}+a_{1}(u) M_{3}\right)\left|x(u)-x^{*}(u)\right|\right\} d u \\
& +a_{2}(t) M_{1}\left|y_{2}\left(t-\tau_{2}\right)-y_{2}^{*}\left(t-\tau_{2}\right)\right|+a_{2}(t) M_{3}\left|x\left(t-\tau_{2}\right)-x^{*}\left(t-\tau_{2}\right)\right| \\
& -r_{1}(t)\left|y_{1}(t)-y_{1}^{*}(t)\right|-r_{2}(t)\left|y_{2}(t)-y_{2}^{*}(t)\right| .
\end{aligned}
$$


Define

$$
\begin{aligned}
V_{2}(t)= & M_{1} \int_{t}^{t+\tau_{1}} \int_{s-\tau_{1}}^{t} a(s)\left\{a(u)\left|x\left(u-\tau_{1}\right)-x^{*}\left(u-\tau_{1}\right)\right|+a_{1}(u)\left|y_{2}(u)-y_{2}^{*}(u)\right|\right. \\
& \left.+\left(r(u)+a(u) M_{1}+a_{1}(u) M_{3}\right)\left|x(u)-x^{*}(u)\right|\right\} d u d s \\
& +M_{1} \int_{t-\tau_{2}}^{t} a_{2}\left(s+\tau_{2}\right)\left|y_{2}(s)-y_{2}^{*}(s)\right| d s+M_{3} \int_{t-\tau_{2}}^{t} a_{2}\left(s+\tau_{2}\right)\left|x(s)-x^{*}(s)\right| d s .
\end{aligned}
$$

Then it follows from (3.6) and (3.7) that for $t>T+2 \tau$,

$$
\begin{aligned}
D^{+} V_{1}(t)+\dot{V}_{2}(t) \leq & -a(t)\left|x(t)-x^{*}(t)\right|+a_{1}(t)\left|y_{2}(t)-y_{2}^{*}(t)\right| \\
& +M_{1} \int_{t}^{t+\tau_{1}} a(s) d s\left\{a(t)\left|x\left(t-\tau_{1}\right)-x^{*}\left(t-\tau_{1}\right)\right|+a_{1}(t)\left|y_{2}(t)-y_{2}^{*}(t)\right|\right. \\
& \left.\quad+\left(r(t)+a(t) M_{1}+a_{1}(t) M_{3}\right)\left|x(t)-x^{*}(t)\right|\right\} \\
& +a_{2}\left(t+\tau_{2}\right) M_{1}\left|y_{2}(t)-y_{2}^{*}(t)\right|+a_{2}\left(t+\tau_{2}\right) M_{3}\left|x(t)-x^{*}(t)\right| \\
& -r_{1}(t)\left|y_{1}(t)-y_{1}^{*}(t)\right|-r_{2}(t)\left|y_{2}(t)-y_{2}^{*}(t)\right| .
\end{aligned}
$$

Let

$$
V(t)=V_{1}(t)+V_{2}(t)+V_{3}(t)
$$

where

$$
V_{3}(t)=M_{1} \int_{t-\tau_{1}}^{t} \int_{s+\tau_{1}}^{s+2 \tau_{1}} a\left(s+\tau_{1}\right) a(u)\left|x(s)-x^{*}(s)\right| d u d s
$$

We derive from (3.8), (3.9), and (3.10) that for $t>T+2 \tau$,

$$
D^{+} V(t) \leq-A_{1}(t)\left|x(t)-x^{*}(t)\right|-r_{1}(t)\left|y_{1}(t)-y_{1}^{*}(t)\right|-A_{2}(t)\left|y_{2}(t)-y_{2}^{*}(t)\right|
$$

where $A_{1}(t)$ and $A_{2}(t)$ are defined in (3.1).

By assumption (H5), there exist constants $\alpha_{1}>0, \alpha_{2}>0$, and a $T^{*} \geq T+2 \tau$ such that for $t \geq T^{*}$,

$$
A_{1}(t) \geq \alpha_{1}>0, \quad A_{2}(t) \geq \alpha_{2}>0 .
$$

Integrating both sides of (3.11) on interval [ $\left.T^{*}, t\right]$, it follows that for $t \geq T^{*}$,

$$
\begin{aligned}
V(t) & +\int_{T^{*}}^{t} A_{1}(s)\left|x(s)-x^{*}(s)\right| d s+\int_{T^{*}}^{t} r_{1}(s)\left|y_{1}(s)-y_{1}^{*}(s)\right| \\
& +\int_{T^{*}}^{t} A_{2}(s)\left|y_{2}(s)-y_{2}^{*}(s)\right| d s \leq V\left(T^{*}\right)
\end{aligned}
$$


We derive from (3.12) and (3.13) that for $t>T^{*}$,

$$
\begin{aligned}
V(t) & +\alpha_{1} \int_{T^{*}}^{t}\left|x(s)-x^{*}(s)\right| d s+r_{1}^{L} \int_{T^{*}}^{t}\left|y_{1}(s)-y_{1}^{*}(s)\right| d s \\
& +\alpha_{2} \int_{T^{*}}^{t}\left|y_{2}(s)-y_{2}^{*}(s)\right| d s \leq V\left(T^{*}\right) .
\end{aligned}
$$

Therefore, $V(t)$ is bounded on $\left[T^{*}, \infty\right)$ and also

$$
\begin{aligned}
& \int_{T^{*}}^{\infty}\left|x(s)-x^{*}(s)\right| d s<\infty, \\
& \int_{T^{*}}^{t}\left|y_{1}(s)-y_{1}^{*}(s)\right| d s<\infty, \\
& \int_{T^{*}}^{\infty}\left|y_{2}(s)-y_{2}^{*}(s)\right| d s<\infty .
\end{aligned}
$$

By Lemma 2.3, $\left|x(t)-x^{*}(t)\right|,\left|y_{1}(t)-y_{1}^{*}(t)\right|$, and $\left|y_{2}(t)-y_{2}^{*}(t)\right|$ are bounded on $\left[T^{*}, \infty\right)$.

On the other hand, it is easy to see that $\dot{x}(t), \dot{x}^{*}(t), \dot{y}_{i}(t)$, and $\dot{y}_{i}^{*}(t)(i=1,2)$ are bounded for $t \geq T^{*}$. Therefore, $\left|x(t)-x^{*}(t)\right|,\left|y_{1}(t)-y_{1}^{*}(t)\right|$, and $\left|y_{2}(t)-y_{2}^{*}(t)\right|$ are uniformly continuous on $\left[T^{*}, \infty\right)$. By Barbalat's lemma [6, Lemmas 1.2.2 and 1.2.3], we conclude that

$$
\lim _{t \rightarrow \infty}\left|x(t)-x^{*}(t)\right|=0, \quad \lim _{t \rightarrow \infty}\left|y_{i}(t)-y_{i}^{*}(t)\right|=0 \quad(i=1,2) .
$$

This completes the proof.

\section{Discussion}

In this paper, we incorporated the periodicity of the ecological and environmental parameters, stage structure for the predator, and time delays due to negative feedback of the prey and due to gestation of the mature predator into a Lotka-Volterra-type predatorprey system. By using Gaines and Mawhin's continuation theorem of coincidence degree theory, we have shown the existence of positive periodic solutions of system (1.1). By proving the permanence of system (1.1) and by using an existence result from [10], we established a set of different sufficient conditions to guarantee the existence of positive periodic solutions to system (1.1). By constructing an appropriate Lyapunov functional, sufficient conditions are derived to confirm that if system (1.1) admits a positive periodic solution, then it is globally stable. By Theorem 2.4, we see that if the prey intrinsic growth rate, the conversion rate of the mature predator, and the rate of immature predator becoming mature predator are high, if the death rates of both the immature and mature predators and the capturing rate of the mature predator are low, and if the time delay due to negative feedback of the prey is small enough, satisfying (H3), (H4), then system (1.1) admits at least one positive periodic solution with the same period as the ecological and environmental parameters. We note that in our result in Theorem 2.2, time delays due to negative feedback of the prey population and due to gestation of the mature predator have no influence on the existence of positive periodic solutions to system (1.1). However, in Theorem 2.4, we need time delay $\tau_{1}$ small enough, satisfying $(\mathrm{H} 3)$. 


\section{Acknowledgment}

The authors would like to thank the referees for their many valuable comments and suggestions that improved the presentation of this work.

\section{References}

[1] W. G. Aiello and H. I. Freedman, A time-delay model of single-species growth with stage structure, Math. Biosci. 101 (1990), no. 2, 139-153.

[2] W. G. Aiello, H. I. Freedman, and J. Wu, Analysis of a model representing stage-structured population growth with state-dependent time delay, SIAM J. Appl. Math. 52 (1992), no. 3, 855-869.

[3] J. M. Cushing, Periodic time-dependent predator-prey systems, SIAM J. Appl. Math. 32 (1977), no. 1, 82-95.

[4] R. E. Gaines and J. L. Mawhin, Coincidence Degree, and Nonlinear Differential Equations, Springer-Verlag, Berlin, 1977.

[5] B. S. Goh, Global stability in two species interactions, J. Math. Biol. 3 (1976), no. 3-4, 313-318.

[6] K. Gopalsamy, Stability and Oscillations in Delay Differential Equations of Population Dynamics, Mathematics and Its Applications, vol. 74, Kluwer Academic Publishers Group, Dordrecht, 1992.

[7] J. Hale, Theory of Functional Differential Equations, Springer-Verlag, New York, 1977.

[8] A. Hastings, Global stability of two-species systems, J. Math. Biol. 5 (1977/78), no. 4, 399-403.

[9] X. He, Stability and delays in a predator-prey system, J. Math. Anal. Appl. 198 (1996), no. 2, 355-370.

[10] Z. Teng and L. Chen, Positive periodic solutions of periodic Kolmogorov type systems with delays, Acta Math. Appl. Sinica 22 (1999), no. 3, 446-456.

[11] W. Wang and L. Chen, A predator-prey system with stage-structure for predator, Comput. Math. Appl. 33 (1997), no. 8, 83-91.

[12] W. Wang and Z. Ma, Harmless delays for uniform persistence, J. Math. Anal. Appl. 158 (1991), no. $1,256-268$.

Rui Xu: Department of Mathematics, Institute of Shijiazhuang Mechanical Engineering, Shijiazhuang 050003, Hebei Province, China

Current address: Department of Mathematics, University of Dundee, Dundee, DD1 4HN, UK

E-mail address: ruixu@maths.dundee.ac.uk

M. A. J. Chaplain: Department of Mathematics, University of Dundee, Dundee, DD1 4HN, UK

E-mail address: chaplain@maths.dundee.ac.uk

F. A. Davidson: Department of Mathematics, University of Dundee, Dundee, DD1 4HN, UK

E-mail address: fdavidso@maths.dundee.ac.uk 


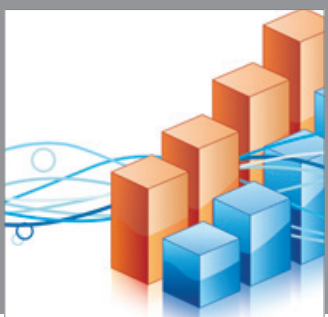

Advances in

Operations Research

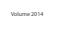

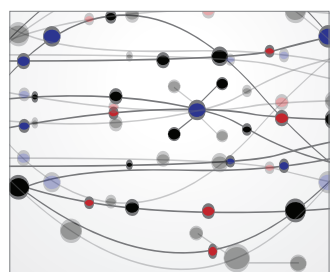

\section{The Scientific} World Journal
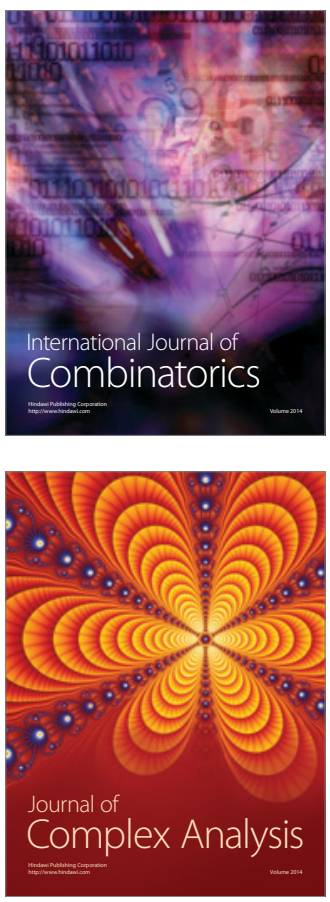

International Journal of

Mathematics and

Mathematical

Sciences
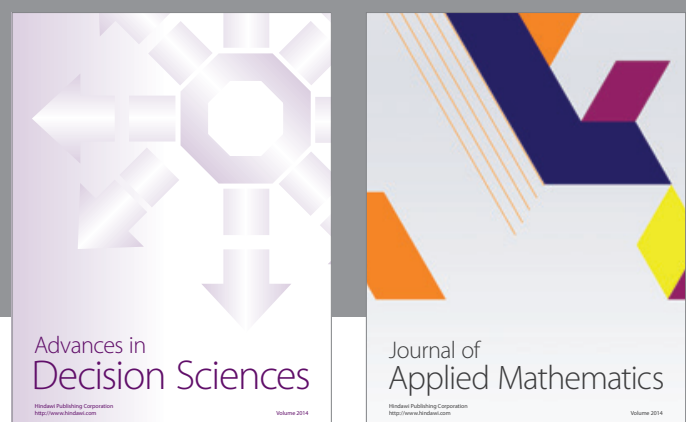

Journal of

Applied Mathematics
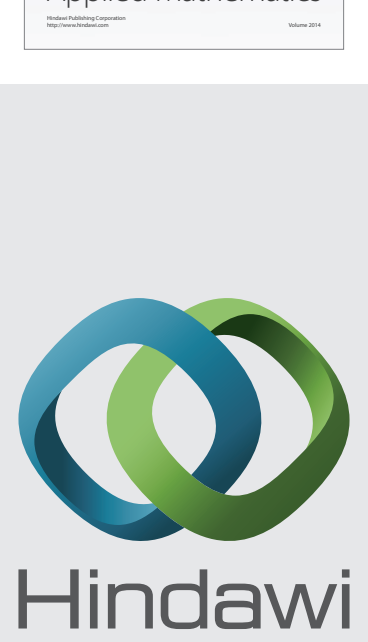

Submit your manuscripts at http://www.hindawi.com
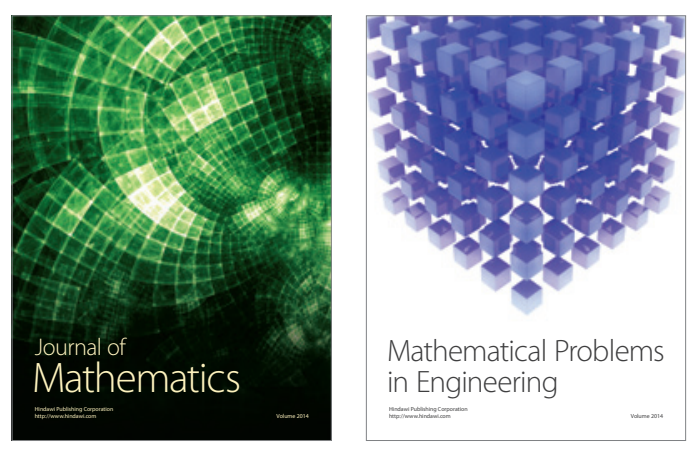

Mathematical Problems in Engineering
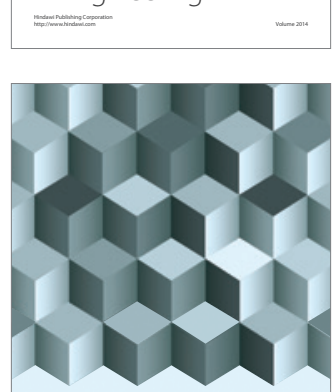

Journal of

Function Spaces
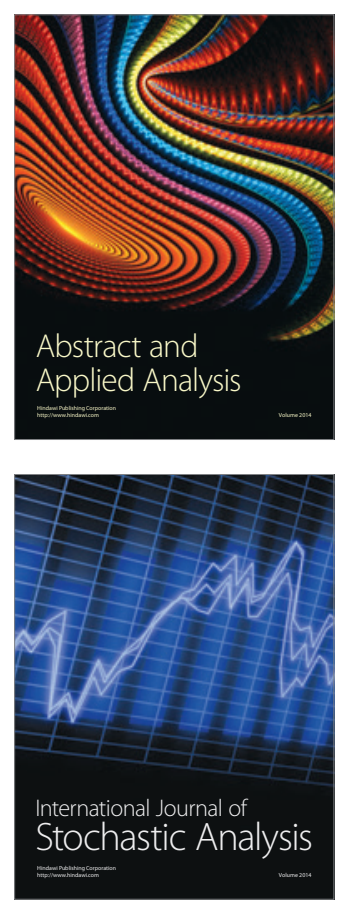

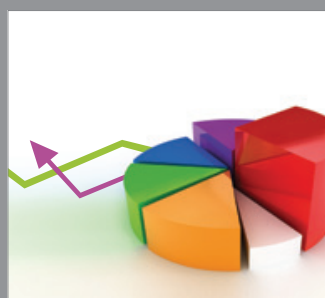

ournal of

Probability and Statistics

Promensencen
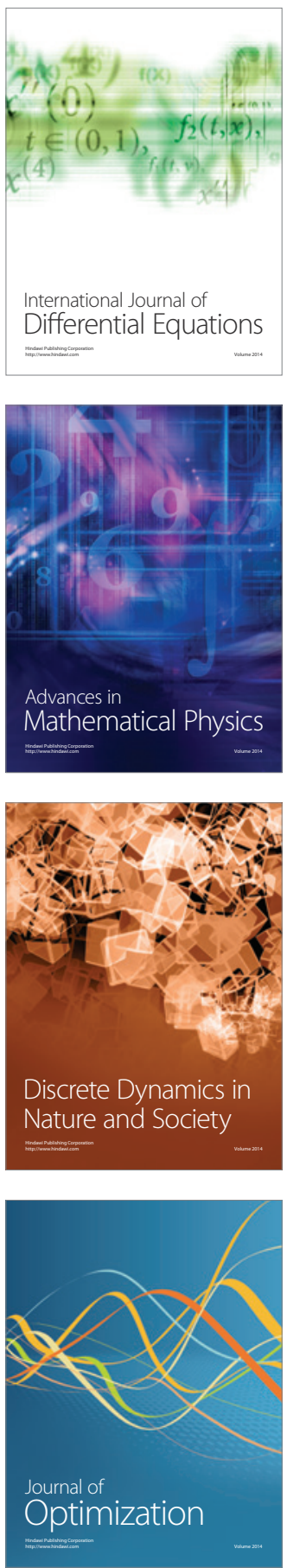